\title{
Focused beam dosimetry of short VHEE bunches
}

\author{
Enrico Brunettia ${ }^{\mathrm{a}}$ Antoine Maitrallain ${ }^{\mathrm{a}}$, and Dino A. Jaroszynski ${ }^{\mathrm{a}}$

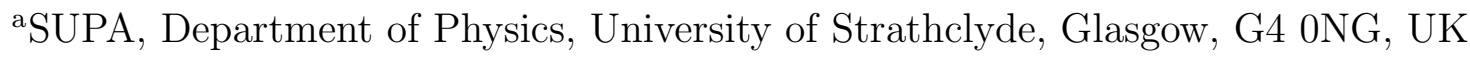

\begin{abstract}
Accelerators driven by 10s TW-class lasers can produce electron bunches with femtosecond-scale duration and energy of 100s of MeV. A potential application of such short bunches is high-dose rate radiotherapy, which could transition to FLASH radiotherapy if a sufficiently large dose is delivered in a single shot. Here we present Monte Carlo simulations to study the bunch length evolution of an electron beam propagating in a water phantom. We show that for electron energies above $100 \mathrm{MeV}$ the bunch lengthens to 1-10 ps duration after interaction with a $30 \mathrm{~cm}$ long water phantom, both for a collimated and weakly focused geometry. The corresponding dose rates are on the order of $200 \mathrm{~Gy} / \mathrm{s}$ per primary electron, much higher than in conventional radiotherapy.
\end{abstract}

Keywords: focused electrons, radiotherapy

\section{INTRODUCTION}

Laser-wakefield accelerators produce electron beams with energy from tens of $\mathrm{MeV}$ to almost $10 \mathrm{GeV}^{1-5}$ in centimetre-scale distances. Simulations and experiments have shown that the duration of these beams is on the order of femtoseconds, ${ }^{6,7}$ much shorter than what is delivered by most conventional radio-frequency accelerators. A possible application of laser-driven accelerators is radiotherapy using very high energy electrons (VHEEs). ${ }^{8,9}$ Because of the short bunch duration, this results in dose delivery at rates much higher than in conventional radiotherapy. ${ }^{10}$ Interest in this regime has been renewed by recent developments in FLASH radiotherapy, ${ }^{11}$ where therapeutical doses are delivered in durations shorter than a few milliseconds, resulting in dose rates in excess of $40 \mathrm{~Gy} / \mathrm{s}$.

Electron beams propagating in a material are subject to scattering, which results in degradation of the beam quality. Lateral scattering can be reduced by increasing the electron energy and by focusing the beam using magnets, ${ }^{12,14}$ resulting in high dose concentration at depth. Here we present results of Monte Carlo simulations to study the bunch length evolution in a water phantom for both collimated and focused beams, investigating the dependence on electron energy and initial bunch duration.

\section{RESULTS}

We performed simulations with the Monte Carlo code Geant $4^{13}$ to model the propagation of electron beams with different energies and bunch durations in a $300 \mathrm{~mm} \times 300 \mathrm{~mm} \times 300 \mathrm{~mm}$ water phantom. It has recently been demonstrated theoretically ${ }^{12}$ and experimentally ${ }^{14}$ that focused VHEE beams can improve dose delivery to deep seated tumours. Therefore, simulations were carried out both for a collimated beam and for a weakly focused beam, using the CLEAR beam line ${ }^{15}$ at CERN as a reference. The beam transport was optimised using MAD-X ${ }^{16}$ for an electron beam with energy of $200 \mathrm{MeV}, 0.1 \%$ energy spread, r.m.s. divergence of $1 \mathrm{mrad}$ and normalised emittance of $1 \pi \mathrm{mm}$ mrad passing through two triplets of quadrupole magnets, as shown in Fig. 1. The beam was focused at the centre of the phantom (in vacuum) with $f$-number $\approx f / 18$, assuming a beam diameter $2 \sigma_{r}$, with $\sigma_{r}$ the r.m.s. radius. The magnetic field gradients are presented in table 1 . The effective length of all quadrupoles was $226 \mathrm{~mm}$, matching the specifications of the CLEAR beam line, but the aperture radius of the final two quadrupoles (Q515 and Q520) was increased to $250 \mathrm{~mm}$ to focus the beam in both planes without clipping. An aperture radius of $150 \mathrm{~mm}$ would have produced similar results, but with a charge loss of about $1 \%$. Even with no clipping, the large quadrupole aperture and relatively short length result in a square beam profile in the horizontal plane at the entrance of the phantom. Here, we assumed hard-edged quadrupoles

Correspondence can be sent to DAJ. E-mail: d.a.jaroszynski@strath.ac.uk 
with no fringe fields, but simulations performed using GPT $^{17}$ with more realistic field profiles produced similar beam shapes. We did not optimise the positions and lengths of the quadrupoles, since these simulations are not intended to model a real experiment, but only to provide insight on the bunch evolution in a general case. In addition to a focusing geometry, we also performed simulations for a collimated beam with an r.m.s. radius of $5 \mathrm{~mm}$.

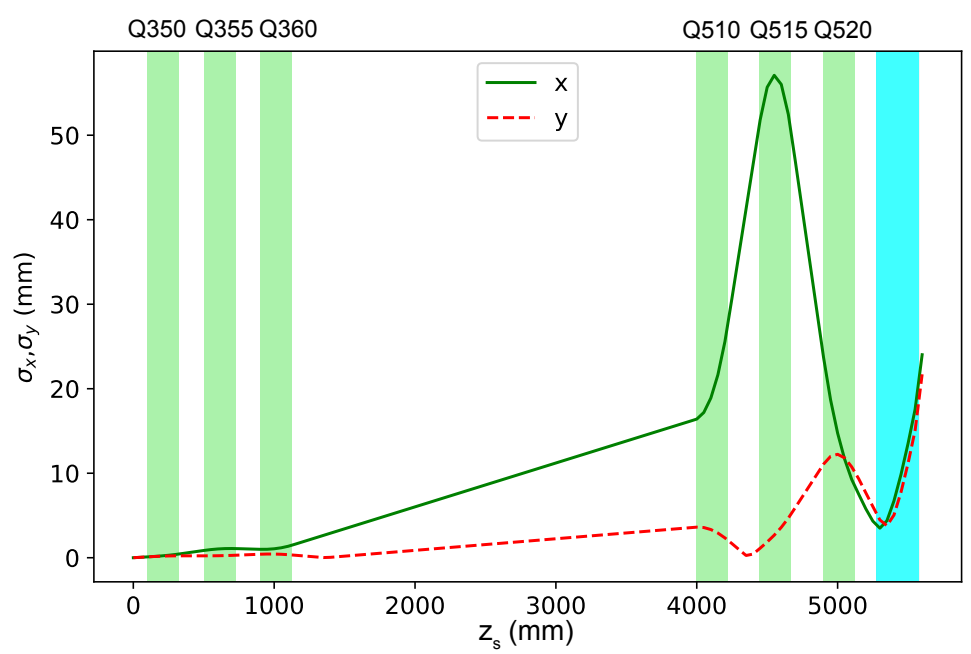

Figure 1: Electron beam transport through a beam line based on the CLEAR facility at CERN. Curves show the r.m.s. beam size in the horizontal $(x)$ and vertical $(y)$ plane for a $200 \mathrm{MeV}$ beam with initial r.m.s. divergence of $1 \mathrm{mrad}$ and normalised emittance of $1 \pi \mathrm{mm}$ mrad focused (in vacuum) at the centre of a water phantom, marked by the blue shaded area. Green shaded areas mark the electromagnetic quadrupoles.

\begin{tabular}{ll} 
Quadrupole & Gradient $(\mathrm{T} / \mathrm{m})$ \\
\hline Q350 & 16.4 \\
Q355 & -8.3 \\
Q360 & 14.8 \\
Q510 & 14.4 \\
Q515 & -11.7 \\
Q520 & 14.9
\end{tabular}

Table 1: Quadrupole gradients used for the simulations with a focused electron beam.

Fig. 2 shows the full-width-at-half-maximum (FWHM) size of a $200 \mathrm{MeV}$ electron beam as it propagates inside the phantom. For a focused geometry (Fig. 2a), the beam size at the phantom entrance $(z=0)$ is about $10 \mathrm{~mm}$, gradually decreasing to a minimum of about $5 \mathrm{~mm}$ at a depth of $100 \mathrm{~mm}$, about $50 \mathrm{~mm}$ upstream from the focus position in vacuum. For a collimated beam (Fig. 2b), the size remains approximately constant for the first $100 \mathrm{~mm}$, until scattering causes it to diverge. The corresponding dose profiles are shown in Fig. 3 for a focused geometry and in Fig. 4 for a collimated beam. In both cases, the highest doses are registered in the first half of the phantom, but a focusing geometry enables to concentrate dose deposition in a small volume at larger depths. For the focusing configuration used here, the dose distribution is slightly asymmetric, as shown by the two-dimensional cross-sections in the horizontal $(z x)$ and vertical $(z y)$ planes presented in Fig. 3a and Fig. 3b, respectively. The square beam profile causes sharp edges to appear in the horizontal plane, but these are gradually smoothed away by interaction with water. The on-axis depth-dose distribution calculated in a $2 \mathrm{~mm} \times 2 \mathrm{~mm} \times 300 \mathrm{~mm}$ volume is presented in Fig. 3c, which is characterised by a peak at a depth of $81 \mathrm{~mm}$ with amplitude of $8.8 \times 10^{-10}$ Gy per primary electron, corresponding to an enhancement of about 4.4 with respect to the surface dose. The corresponding depth-dose profile generated by a collimated beam (Fig. 4c), on the other hand, is approximately flat for the first $60 \mathrm{~mm}$, with a dose of about $2 \times 10^{-10}$ Gy per primary electron. The depth-dose profile calculated in a $100 \mathrm{~mm} \times 100 \mathrm{~mm} \times 300 \mathrm{~mm}$ volume centred on-axis is similar for both 
a

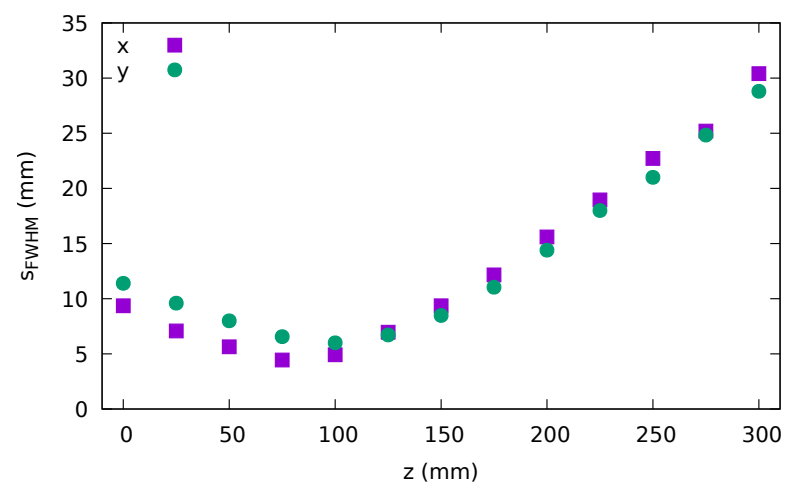

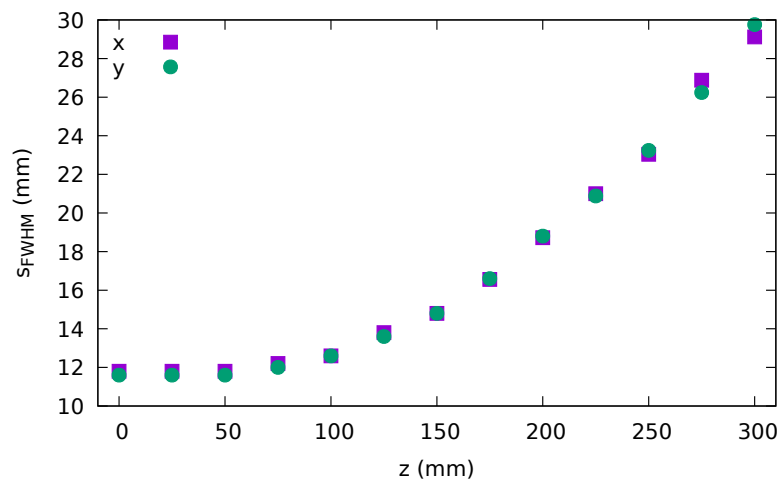

Figure 2: Transverse size (FWHM) versus depth in the phantom of $\mathbf{a}$ a focused and $\mathbf{b}$ a collimated electron beam with energy of $200 \mathrm{MeV}$.

a
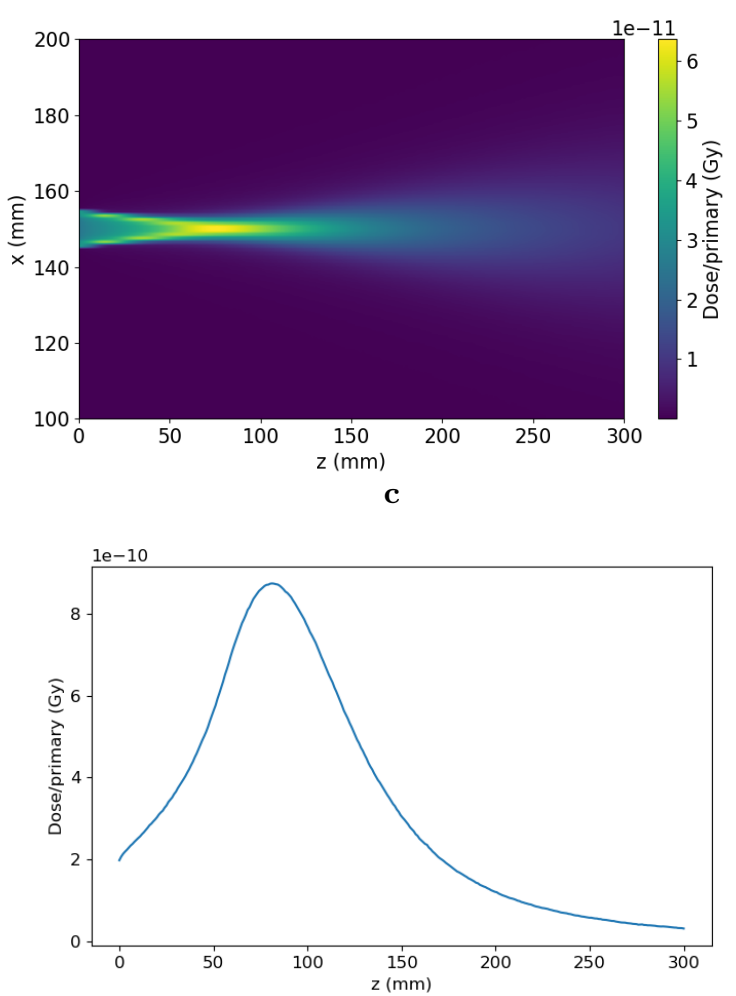

b
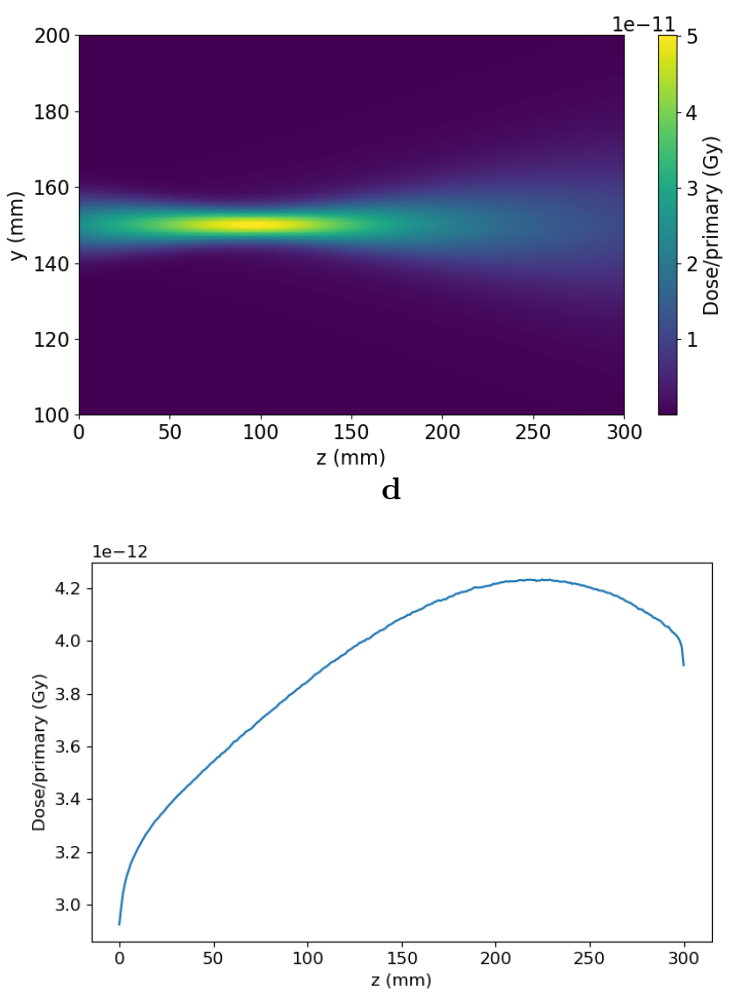

Figure 3: Dose deposited in the phantom by a $200 \mathrm{MeV}$ focused electron beam. a Two-dimensional distribution in the $z x$ plane, calculated in a $2 \mathrm{~mm} \times 100 \mathrm{~mm} \times 300 \mathrm{~mm}$ volume. b Two-dimensional distribution in the $z y$ plane, calculated in a $100 \mathrm{~mm} \times 2 \mathrm{~mm} \times 300 \mathrm{~mm}$ volume. c On-axis depth-dose profile calculated in a $2 \mathrm{~mm} \times$ $2 \mathrm{~mm} \times 300 \mathrm{~mm}$ volume. $\mathbf{d}$ Depth-dose profile calculated in a $100 \mathrm{~mm} \times 100 \mathrm{~mm} \times 300 \mathrm{~mm}$ volume. 

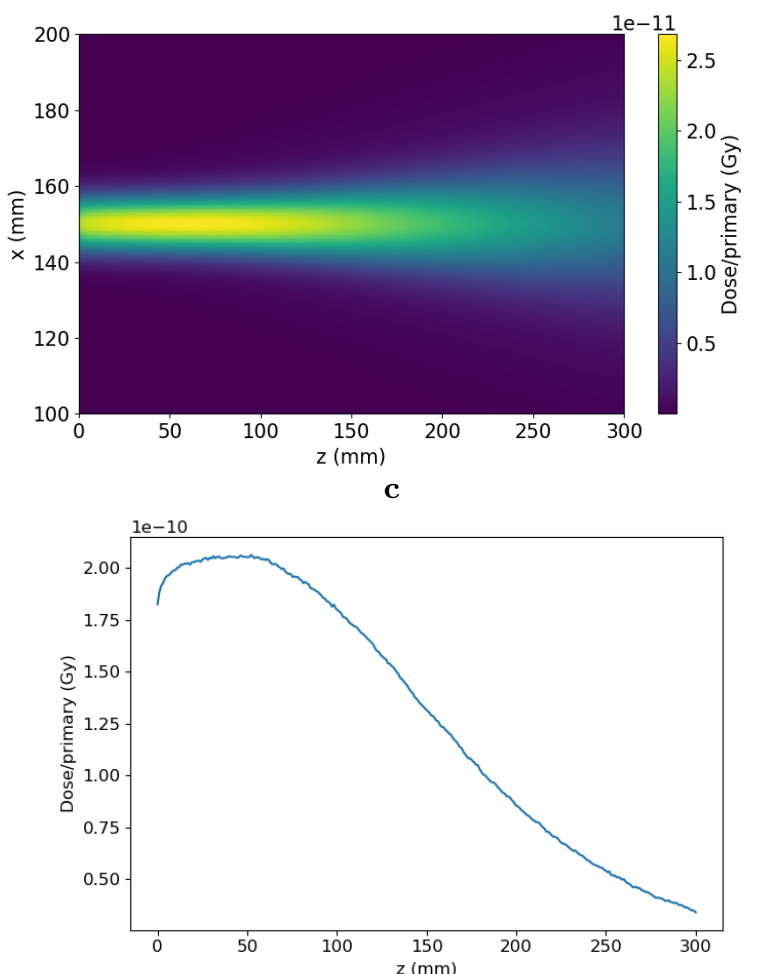

b
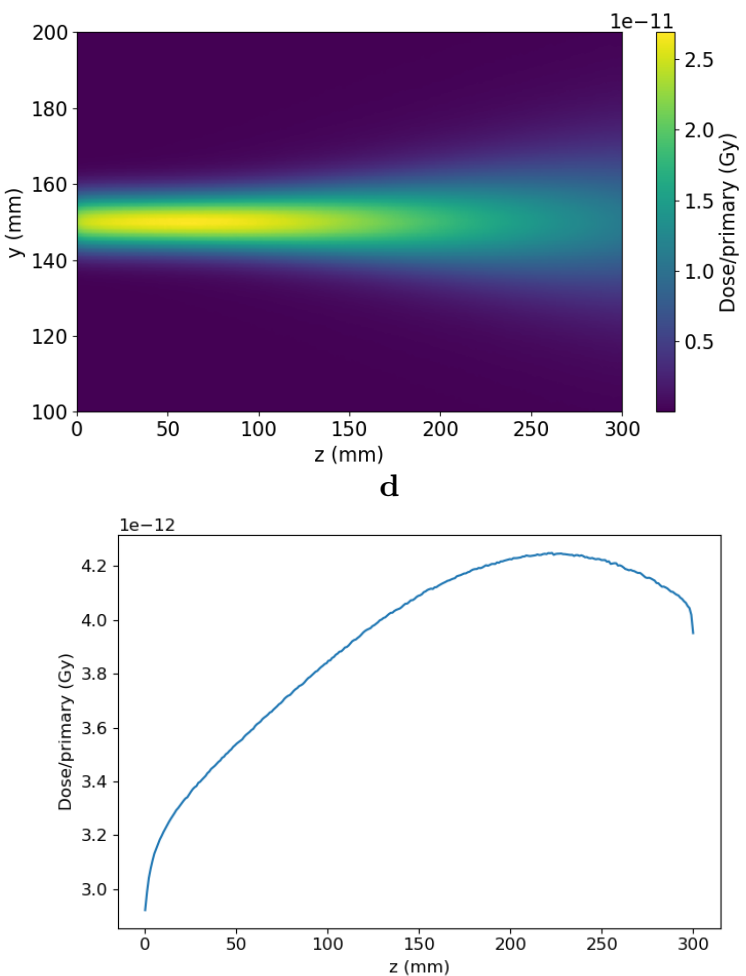

Figure 4: Dose deposited in the phantom by a $200 \mathrm{MeV}$ collimated electron beam with r.m.s. size of $5 \mathrm{~mm}$. a Twodimensional distribution in the $z x$ plane, calculated in a $2 \mathrm{~mm} \times 100 \mathrm{~mm} \times 300 \mathrm{~mm}$ volume. b Two-dimensional distribution in the $z y$ plane, calculated in a $100 \mathrm{~mm} \times 2 \mathrm{~mm} \times 300 \mathrm{~mm}$ volume. c On-axis depth-dose profile calculated in a $2 \mathrm{~mm} \times 2 \mathrm{~mm} \times 300 \mathrm{~mm}$ volume. $\mathbf{d}$ Depth-dose profile calculated in a $100 \mathrm{~mm} \times 100 \mathrm{~mm} \times 300 \mathrm{~mm}$ volume.

geometries, as shown in Fig. 3d and Fig. 4d.

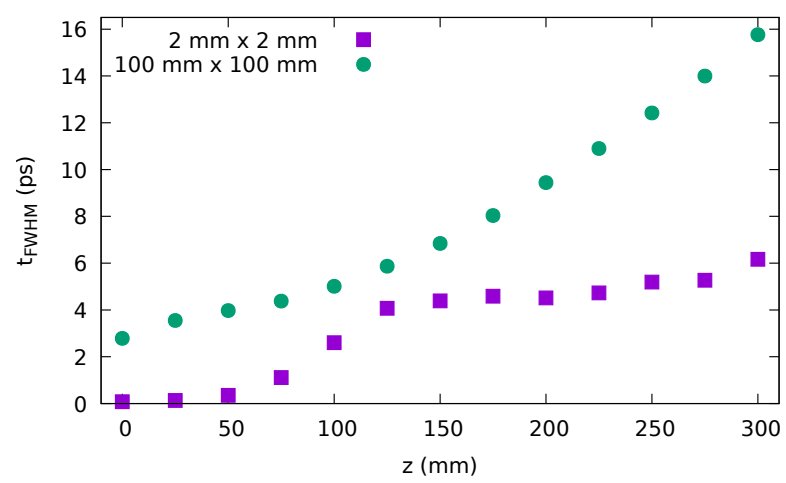

b

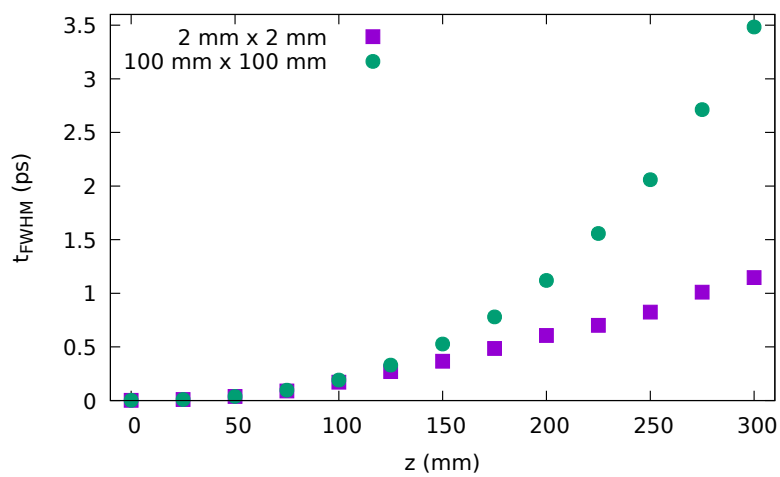

Figure 5: Bunch duration at different depths in the phantom for an electron beam with $200 \mathrm{MeV}$ energy and initial r.m.s. bunch duration of 1 fs. a Focused geometry. b A collimated beam with 5 mm r.m.s. size.

The evolution of the bunch duration inside the phantom for an electron beam with $200 \mathrm{MeV}$ energy and initial r.m.s. duration of $1 \mathrm{fs}$ and 1 ps is shown in Fig. 5 and Fig. 6, respectively. Only electrons propagating in the forward direction with momentum $p_{z}>1 \mathrm{MeV} / \mathrm{c}$ are included. For a focused geometry, electrons converge 


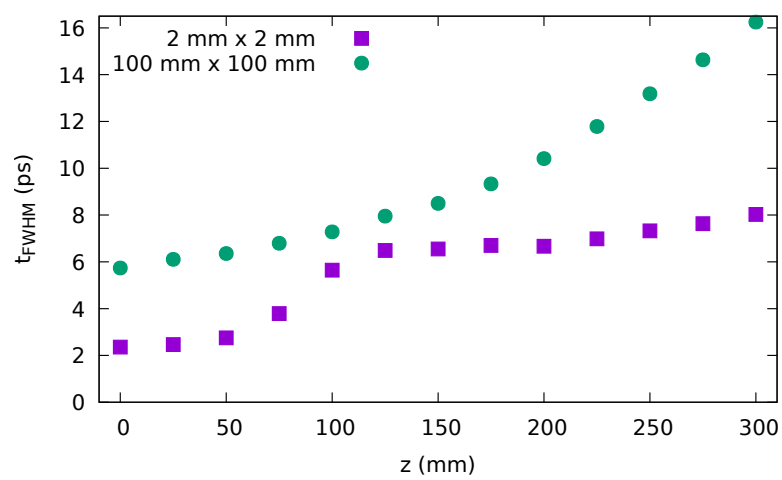

b

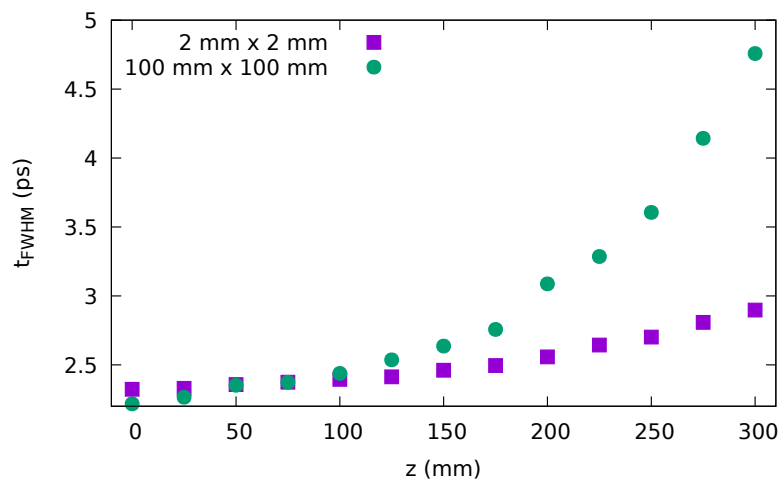

Figure 6: Bunch duration at different depths in the phantom for an electron beam with $200 \mathrm{MeV}$ energy and initial r.m.s. bunch duration of 1 ps. a Focused geometry. b A collimated beam with 5 mm r.m.s. size.

towards the centre of the phantom after travelling along different trajectories, resulting in larger path differences and longer bunch durations than for a collimated beam. For an initial r.m.s. bunch duration of $1 \mathrm{fs}(2.35 \mathrm{fs}$ FWHM), the FWHM bunch duration at the centre of the phantom $(z=150 \mathrm{~mm})$ calculated in a $2 \mathrm{~mm} \times 2 \mathrm{~mm}$ cross-sectional area centred on-axis is about $4.5 \mathrm{ps}$, in contrast to a $0.35 \mathrm{ps}$ duration obtained for a collimated beam. The bunch duration calculated in a $100 \mathrm{~mm} \times 100 \mathrm{~mm}$ area is about $7 \mathrm{ps}$ for a focused beam and $0.5 \mathrm{ps}$ for a collimated beam. If the initial r.m.s. bunch duration is 1 ps (2.35 ps FWHM), on the other hand, the FWHM bunch duration at the centre of the phantom is about $6.5 \mathrm{ps}$ for a focused beam and $2.5 \mathrm{ps}$ for a collimated beam when using a $2 \mathrm{~mm} \times 2 \mathrm{~mm}$ area, growing to $8.5 \mathrm{ps}$ and $2.6 \mathrm{ps}$, respectively, in a $100 \mathrm{~mm} \times 100 \mathrm{~mm}$ area.

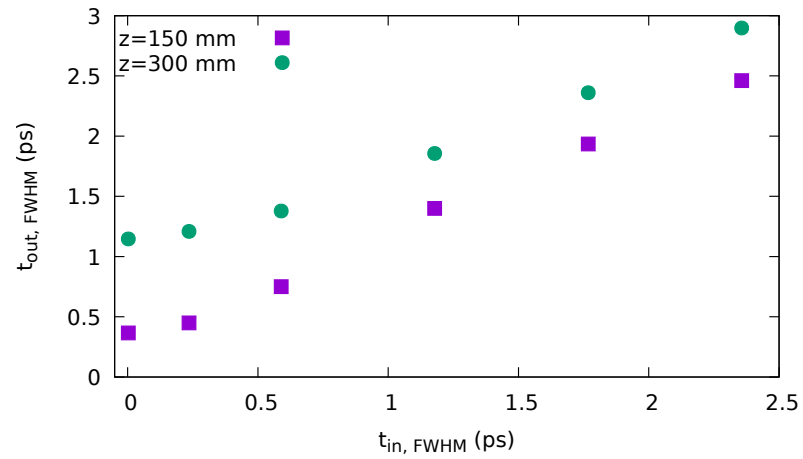

b

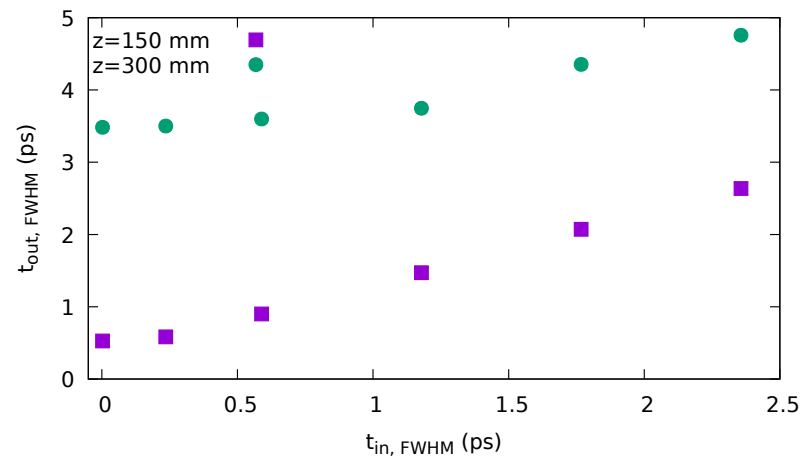

Figure 7: Bunch duration at the centre $(z=150 \mathrm{~mm})$ and exit $(z=300 \mathrm{~mm})$ of the phantom calculated a in a $2 \mathrm{~mm} \times 2 \mathrm{~mm}$ cross-sectional area and $\mathbf{b}$ in a $100 \mathrm{~mm} \times 100 \mathrm{~mm}$ cross-sectional area. The electron beam is collimated and has energy of $200 \mathrm{MeV}$, initial r.m.s. size of $5 \mathrm{~mm}$ and varying initial bunch duration.

The dependence of bunch lengthening on the initial bunch duration is shown in Fig. 7 for a collimated electron beam with energy of $200 \mathrm{MeV}$. When the initial bunch duration is on the order of femtoseconds or tens of femtoseconds, bunch lengthening in water is large. For example, a bunch with r.m.s duration of 1 fs (2.35 fs FWHM) lengthens by a factor of about 150 after $150 \mathrm{~mm}$ propagation in water and by a factor of 500 after $300 \mathrm{~mm}$, when considering a $2 \mathrm{~mm} \times 2 \mathrm{~mm}$ area. For bunch durations on the order of $100 \mathrm{~s}$ of femtoseconds or longer, on the other hand, bunch lengthening is small. For example, a bunch with r.m.s. duration of $250 \mathrm{fs}$ ( $590 \mathrm{fs}$ FWHM) lengthens by a factor of 1.3 after $150 \mathrm{~mm}$ and 2.3 after $500 \mathrm{~mm}$.

The dependence of bunch lengthening on the electron beam energy is shown in Fig. 8 for a collimated beam with initial r.m.s. duration of $1 \mathrm{fs}$. The bunch length increases steeply at low electron energies. For example, for an energy of $100 \mathrm{MeV}$ the bunch lengthens by a factor of about 350 after $150 \mathrm{~mm}$ propagation in water and by a factor of 2400 at the exit of the phantom, when considering a $2 \mathrm{~mm} \times 2 \mathrm{~mm}$ cross-sectional area. In 


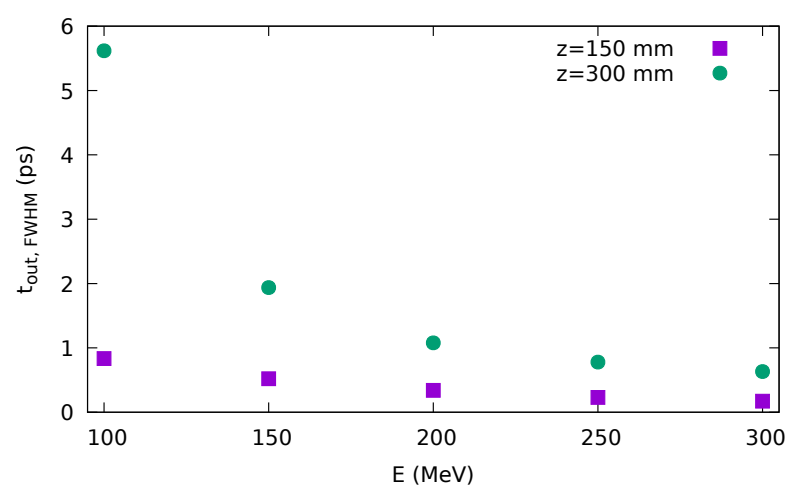

b

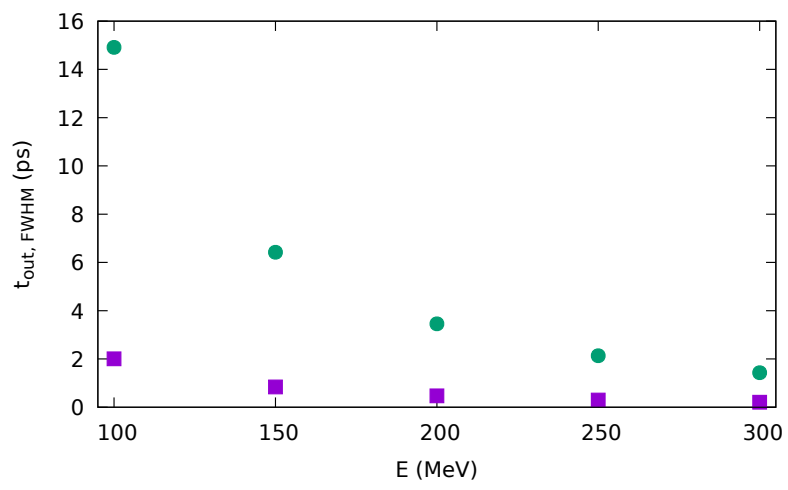

Figure 8: Bunch duration at the centre $(z=150 \mathrm{~mm})$ and exit $(z=300 \mathrm{~mm})$ of the phantom calculated a in a $2 \mathrm{~mm} \times 2 \mathrm{~mm}$ cross-sectional area and $\mathbf{b}$ in a $100 \mathrm{~mm} \times 100 \mathrm{~mm}$ cross-sectional area. The electron beam is collimated and has energy between $100 \mathrm{MeV}$ and $300 \mathrm{MeV}$, initial r.m.s. size of $5 \mathrm{~mm}$ and initial r.m.s. bunch duration of $1 \mathrm{fs}$.

a $100 \mathrm{~mm} \times 100 \mathrm{~mm}$ area, the bunch lengthens by a factor of 850 and 6300 , respectively. For energies above $200 \mathrm{MeV}$, on the other hand, the bunch lengthening slowly decreases with energy.

\section{DISCUSSION}

We investigated the propagation of VHHE beams in water for collimated and focused geometries. We have shown that the typical bunch durations in the middle of a $300 \mathrm{~mm} \times 300 \mathrm{~mm} \times 300 \mathrm{~mm}$ phantom are close to 1 ps for electron beams with $200 \mathrm{MeV}$ energy and initial bunch duration between $1 \mathrm{fs}$ and 1 ps. Assuming an accelerator operating at a repetition rate of $1 \mathrm{~Hz}$, the corresponding dose rates are about $200 \mathrm{~Gy} / \mathrm{s}$ per primary electron, which is many orders of magnitude higher than in conventional radiotherapy. We have shown that focusing the electron beam results in an additional bunch lengthening by a factor between 3 and 10, but the peak dose at depth is enhanced. Typically, laser-driven accelerators produce electron beams with charges of the order of $10 \mathrm{~s}$ or $100 \mathrm{~s}$ of $\mathrm{pC}$, resulting in doses of $10 \mathrm{~s}$ of mGy. These values are expected to be too low to observe FLASH effect, which requires doses of at least 5-10 Gy, based on current knowledge. ${ }^{18}$ Nevertheless, these beams allow to explore regimes that have not been extensively studied so far.

\section{ACKNOWLEDGMENTS}

The authors acknowledge funding from U.K. EPSRC (EP/J018171/1, EP/N028694/1), EU H2020 Research and Innovation Programme LASERLAB EUROPE (871124) and EuPRAXIA (653782). Data associated with research published in this paper is available at https://doi.org/10.15129/39fcbf9d-3062-4744-8c7f-b5b9894ec317.

\section{REFERENCES}

[1] Mangles, S. P. D., Murphy, C. D., Najmudin, Z., Thomas, A. G. R., Collier, J. L., Dangor, A. E., Divall, E. J., Foster, P. S., Gallacher, J. G., Hooker, C. J., Jaroszynski, D. A., Langley, A. J., Mori, W. B., Norreys, P. A., Tsung, F. S., Viskup, R., Walton, B. R., and Krushelnick, K., "Monoenergetic beams of relativistic electrons from intense laser-plasma interactions," Nature 431, 535-538 (Sept. 2004).

[2] Geddes, C. G. R., Toth, C., van Tilborg, J., Esarey, E., Schroeder, C. B., Bruhwiler, D., Nieter, C., Cary, J., and Leemans, W. P., "High-quality electron beams from a laser wakefield accelerator using plasma-channel guiding," Nature 431, 538-541 (Sept. 2004).

[3] Faure, J., Glinec, Y., Pukhov, A., Kiselev, S., Gordienko, S., Lefebvre, E., Rousseau, J.-P., Burgy, F., and Malka, V., "A laser-plasma accelerator producing monoenergetic electron beams," Nature 431, 541-544 (Sept. 2004).

[4] Esarey, E., Schroeder, C. B., and Leemans, W. P., "Physics of laser-driven plasma-based electron accelerators," Reviews of Modern Physics 81, 1229-1285 (Aug. 2009). 
[5] Gonsalves, A. J., Nakamura, K., Daniels, J., Benedetti, C., Pieronek, C., de Raadt, T. C. H., Steinke, S., Bin, J. H., Bulanov, S. S., van Tilborg, J., Geddes, C. G. R., Schroeder, C. B., Tóth, C., Esarey, E., Swanson, K., Fan-Chiang, L., Bagdasarov, G., Bobrova, N., Gasilov, V., Korn, G., Sasorov, P., and Leemans, W. P., "Petawatt Laser Guiding and Electron Beam Acceleration to $8 \mathrm{GeV}$ in a Laser-Heated Capillary Discharge Waveguide," Physical Review Letters 122, 084801 (Feb. 2019).

[6] Lundh, O., Lim, J., Rechatin, C., Ammoura, L., Ben-Ismaïl, A., Davoine, X., Gallot, G., Goddet, J.-P., Lefebvre, E., Malka, V., and Faure, J., "Few femtosecond, few kiloampere electron bunch produced by a laser-plasma accelerator," Nature Physics 7, 219-222 (Mar. 2011).

[7] Islam, M. R., Brunetti, E., Shanks, R. P., Ersfeld, B., Issac, R. C., Cipiccia, S., Anania, M. P., Welsh, G. H., Wiggins, S. M., Noble, A., Cairns, R. A., Raj, G., and D A Jaroszynski, "Near-threshold electron injection in the laser-plasma wakefield accelerator leading to femtosecond bunches," New Journal of Physics 17, 093033 (Sept. 2015).

[8] DesRosiers, C., Moskvin, V., Bielajew, A. F., and Papiez, L., "150-250 MeV electron beams in radiation therapy," Physics in Medicine and Biology 45, 1781-1805 (July 2000).

[9] Nakajima, K., "Laser-driven electron beam and radiation sources for basic, medical and industrial sciences," Proceedings of the Japan Academy, Series B 91(6), 223-245 (2015).

[10] Subiel, A., Moskvin, V., Welsh, G. H., Cipiccia, S., Reboredo, D., Evans, P., Partridge, M., DesRosiers, C., Anania, M. P., Cianchi, A., Mostacci, A., Chiadroni, E., Di Giovenale, D., Villa, F., Pompili, R., Ferrario, M., Belleveglia, M., Di Pirro, G., Gatti, G., Vaccarezza, C., Seitz, B., Isaac, R. C., Brunetti, E., Wiggins, S. M., Ersfeld, B., Islam, M. R., Mendonca, M. S., Sorensen, A., Boyd, M., and Jaroszynski, D. A., "Dosimetry of very high energy electrons (VHEE) for radiotherapy applications: Using radiochromic film measurements and Monte Carlo simulations," Physics in Medicine and Biology 59, 5811-5829 (Oct. 2014).

[11] Hendry, J., "Taking Care with FLASH Radiation Therapy," International Journal of Radiation Oncology*Biology*Physics 107, 239-242 (June 2020).

[12] Kokurewicz, K., Brunetti, E., Welsh, G. H., Wiggins, S. M., Boyd, M., Sorensen, A., Chalmers, A. J., Schettino, G., Subiel, A., DesRosiers, C., and Jaroszynski, D. A., "Focused very high-energy electron beams as a novel radiotherapy modality for producing high-dose volumetric elements," Scientific Reports $\mathbf{9}, 10837$ (Dec. 2019).

[13] Agostinelli, S., Allison, J., Amako, K., Apostolakis, J., Araujo, H., Arce, P., Asai, M., Axen, D., Banerjee, S., Barrand, G., Behner, F., Bellagamba, L., Boudreau, J., Broglia, L., Brunengo, A., Burkhardt, H., Chauvie, S., Chuma, J., Chytracek, R., Cooperman, G., Cosmo, G., Degtyarenko, P., Dell'Acqua, A., Depaola, G., Dietrich, D., Enami, R., Feliciello, A., Ferguson, C., Fesefeldt, H., Folger, G., Foppiano, F., Forti, A., Garelli, S., Giani, S., Giannitrapani, R., Gibin, D., Gómez Cadenas, J., González, I., Gracia Abril, G., Greeniaus, G., Greiner, W., Grichine, V., Grossheim, A., Guatelli, S., Gumplinger, P., Hamatsu, R., Hashimoto, K., Hasui, H., Heikkinen, A., Howard, A., Ivanchenko, V., Johnson, A., Jones, F., Kallenbach, J., Kanaya, N., Kawabata, M., Kawabata, Y., Kawaguti, M., Kelner, S., Kent, P., Kimura, A., Kodama, T., Kokoulin, R., Kossov, M., Kurashige, H., Lamanna, E., Lampén, T., Lara, V., Lefebure, V., Lei, F., Liendl, M., Lockman, W., Longo, F., Magni, S., Maire, M., Medernach, E., Minamimoto, K., Mora de Freitas, P., Morita, Y., Murakami, K., Nagamatu, M., Nartallo, R., Nieminen, P., Nishimura, T., Ohtsubo, K., Okamura, M., O’Neale, S., Oohata, Y., Paech, K., Perl, J., Pfeiffer, A., Pia, M., Ranjard, F., Rybin, A., Sadilov, S., Di Salvo, E., Santin, G., Sasaki, T., Savvas, N., Sawada, Y., Scherer, S., Sei, S., Sirotenko, V., Smith, D., Starkov, N., Stoecker, H., Sulkimo, J., Takahata, M., Tanaka, S., Tcherniaev, E., Safai Tehrani, E., Tropeano, M., Truscott, P., Uno, H., Urban, L., Urban, P., Verderi, M., Walkden, A., Wander, W., Weber, H., Wellisch, J., Wenaus, T., Williams, D., Wright, D., Yamada, T., Yoshida, H., and Zschiesche, D., "Geant4 - a simulation toolkit," Nuclear Instruments and Methods in Physics Research Section A: Accelerators, Spectrometers, Detectors and Associated Equipment 506, 250-303 (July 2003).

[14] Kokurewicz, K., Brunetti, E., Curcio, A., Gamba, D., Garolfi, L., Gilardi, A., Senes, E., Sjobak, K. N., Farabolini, W., Corsini, R., and Jaroszynski, D. A., "An experimental study of focused very high energy electron beams for radiotherapy," Communications Physics 4(1), 33 (2021).

[15] Sjobak, K., Adli, E., Bergamaschi, M., Burger, S., Corsini, R., Curcio, A., Curt, S., Döbert, S., Farabolini, W., Gamba, D., Garolfi, L., Gilardi, A., Gorgisyan, I., Granados, E., Guerin, H., Kieffer, R., Krupa, M., Lefèvre, T., Lindstrøm, C., Lyapin, A., Mazzoni, S., McMonagle, G., Nadenau, J., Panuganti, H., Pitman, 
S., Rude, V., Schlogelhofer, A., Skowroński, P., Wendt, M., and Zemanek, A., "Status of the CLEAR Electron Beam User Facility at CERN," Proceedings of the 10th Int. Particle Accelerator Conf. IPAC2019, 4 pages, $0.190 \mathrm{MB}(2019)$.

[16] Grote, H. and Schmidt, F., "Mad-X - an upgrade from mad8," in [Proceedings of the 2003 Particle Accelerator Conference], 3497-3499, IEEE, Portland, OR (2003).

[17] "General Particle Tracer." http://www.pulsar.nl/gpt.

[18] Vozenin, M.-C., Hendry, J., and Limoli, C., "Biological Benefits of Ultra-high Dose Rate FLASH Radiotherapy: Sleeping Beauty Awoken," Clinical Oncology 31, 407-415 (July 2019). 